\title{
Coloring Characteristic of Lead Glass for X-Ray Irradiation
}

\author{
Michiharu Sekimoto', Yoh Katoh ${ }^{2}$ \\ ${ }^{1}$ Medicine and Medical Sciences, University of Tsukuba, Tsukuba, Japan \\ ${ }^{2}$ School of Radiology, Faculty of Health Sciences, Tokyo Metropolitan University, Hachiōji, Japan \\ Email: sekimoto.m.ff@u.tsukuba.ac.jp
}

Received 24 April 2015; accepted 30 May 2015; published 2 June 2015

Copyright (C) 2015 by authors and Scientific Research Publishing Inc.

This work is licensed under the Creative Commons Attribution International License (CC BY).

http://creativecommons.org/licenses/by/4.0/

c. (i) Open Access

\begin{abstract}
The lead glass used in an X-ray examination room is indispensable for watching a subject being tested or a situation of operation of an X-ray generator, but because it is some structure equipment of an X-ray examination room, it must obey legal restrictions. Although the standard for lead glass is determined by the International Electrotechnical Commission (IEC), there are few reports about the characteristics related to interaction with radiation. We performed a comparison of three lead glass products, which are principally used in world. As for three products, differences are not looked by physics and chemical characteristics features. Simply the difference was looked by the coloring characteristic for an interaction with radiation. Furthermore, we checked that temporal decolorization of coloring had two half-lives. The short half-life is about 28 hours and the long half-life is about 107 days.
\end{abstract}

\section{Keywords}

Lead Glass, Coloring, Absorbance, Half-Life

\section{Introduction}

The standard for the buildings and the facilities for an X-ray examination room are stipulated in reports No. 145 and 147 of National Council of Radiation Protection and Measurements (NCRP) [1] [2], which state that "the ceiling, floor, and partition walls must be capable of shielding such that, outside them, the weekly effective dose is below 1 millisievert”. Therefore, the X-ray protective lead glass used for looking inside the X-ray examination room must also be subject to the regulations, because it forms part of the partition wall. Furthermore, $\mathrm{X}$-ray protective lead glass must conform to the specifications given by the International Electrotechnical Commission (IEC) 61331-2 [3].

Usually, when relatively high-energy electromagnetic waves, such as ultraviolet rays, $\mathrm{X}$-rays, and gamma rays, 
irradiate colorless glass, there is coloration due to 1) generation of color center (brown lines), 2) the generation of micro-particles (yellow lines), and 3) change in the ionic valence (blue-red lines) [4]. The lead glass has been colored with 1) color center. Oxygen bonded to one silicon (called non-bridging oxygen) is generated by the non-bridging oxygen hole centers electrons jumping out in interaction with electromagnetic waves; most of the released electrons return to the original. However, some may be trapped in such an impurity in the glass; a pair of holes and the trapping electrons are generated. This is called a color center, and because it absorbs at around 430 and $620 \mathrm{~nm}$ in the visible wavelength range, it gives the glass a brownish color [5]. The coloration of light-induced origin is reversible, meaning that the glass can be returned to colorless glass by decolorizing with heating at temperatures of $300^{\circ} \mathrm{C}-550^{\circ} \mathrm{C}$ for approximately 30 minutes. However, it is observed some degree of fading even at about room temperature.

A discussion concerning the lead glass used in X-ray examination rooms should include such things: its shielding capability, transparency, coloring, and maintenance, but reports specializing in lead glass cannot be found. Thus, on this occasion, through a comparison of products which have newly entered the X-ray protective lead glass market and earlier products, we will report on our investigations into 1) physical and chemical properties of different types of lead glass, 2) the transparency when not irradiated with X-rays, 3) coloring according to the irradiation level, and, 4) color fading with the passage of time.

\section{The Equipment and Method Used}

The lead glass that was used is a nominal $1.5 \mathrm{~mm} \mathrm{~Pb}$ equivalent of $\mathrm{ZFx}\left(\mathrm{SiO}_{2}: \geq 50\right.$ [Wt\%], $\mathrm{K}_{2} \mathrm{O}: 5-10$ [Wt\%], $\mathrm{As}_{2} \mathrm{O}_{3}: 0$ - 5 [Wt\%], PbO: $\leq 55$ [Wt\%]; Shanghai Sai Fukang Radiation Protection Equipment Co., Ltd. China), MedX (Ba: 17 [\%], Pb: 52 [\%]; Corning Incorporated, USA), LX-57B (Chemical components Unknown; Nippon Electric Glass Co., Ltd. JAPAN). These were cut into $10 \times 50 \mathrm{~mm}$ for measuring the absorbance at absorptiometer U-1900 (Hitachi High-Technologies Co. JAPAN).

The X-ray generator was an ISOVOLT Titan E 160 (General Electric Company. USA), and the X-ray tube was an ISOVOLT $160 \mathrm{M} 2 / 0.4$ - 3.0 with a tungsten target (1 mm Be window). Because the lead glass installed for medical examinations is normally on a side scattered radiation from the subject rather than on a direct radiation, an additional filter was not used.

Setting the tube voltage at $80 \mathrm{kV}$, the tube current at $6 \mathrm{~mA}$, and a $100 \mathrm{~cm}$ distance between the $\mathrm{X}$-ray tube focus and the lead glass, the absorbed dose is $10 \mathrm{mGy} / \mathrm{s}$. For a distance of $100 \mathrm{~cm}$ from an actual subject placed in the examination room to the lead glass (right-angled direction of X-ray beam), under filming conditions for a plain X-ray of the abdomen (at a tube voltage of $80 \mathrm{kV}$ and $20 \mathrm{~mA}$ ), we obtained a measured value of approximately $15 \mu$ Gy per exposure. For 300 exposures in 1 day and 300 operating days in a year, the lead glass is then exposed to approximately 1 Gy per 1 year.

For establishing the extent of the coloring due to the absorbed dose, for lead glass from different companies, the same lead glass was cumulatively irradiated for $0,100,200,300,500,700,1000,1300$, 2000, and 5000 seconds ( 0 - $50 \mathrm{~Gy})$. After completion of each cumulative irradiation, the absorbance at 300 - $900 \mathrm{~nm}$ was immediately measured with an absorptiometer.

To understand to what extent the color will fade with time following fully colored lead glass, each individual lead glass arranged as in 2.3 was irradiated continuously for 15 hours and then measured with an absorptiometer over the same wavelength range as in 2.4 over a 3-day period immediately following the completion of irradiation. The room temperature for both the X-ray examination room and the absorptiometer measuring chamber was adjusted to be $27^{\circ} \mathrm{C}$.

\section{Results and Discussions}

\subsection{Physical and Chemical Properties of Different Types of Lead Glass}

Table 1 shows the content of the main components in ZFx, MedX, and LX-57B [Wt\%]. The composition of ZFx mainly constitutes lead oxide (II) and silicon oxide, containing a small percentage of potassium oxide. On the other hand, MedX and LX-57B contain barium oxide. Although it does not appear in the manufacturer's published tables of ingredients, it is thought that silicon oxide must also be present in the glass, and it can be assumed, as seen in the tables of ingredients, that MedX and LX-57B contain approximately $37 \%$.

Due to the fact that barium (atomic number $\mathrm{Z}=56$ ) has a high atomic number compared to potassium $(\mathrm{Z}=$ 19), the mass energy absorption coefficient $\mu_{e n} / \rho\left[\mathrm{cm}^{2} / \mathrm{g}\right]$ is high (for example, it is 8.875 for barium and 
Table 1. Physical and chemical properties of different type of lead glass.

\begin{tabular}{|c|c|c|c|c|}
\hline & & ZFx & MedX & LX-57B \\
\hline \multicolumn{2}{|c|}{ Lead equivalent $[\mathrm{mmPb}]$} & & $<1.5$ & \\
\hline \multirow{5}{*}{ Main component [Wt\%] } & Lead oxide (II) PbO & $>55$ & $<48$ & 55 \\
\hline & Silicon oxide $\mathrm{SiO}$ & $<50$ & (37) & $(37)$ \\
\hline & Potassium oxide $\mathrm{K}_{2} \mathrm{O}$ & $<10$ & - & - \\
\hline & Barium oxide $\mathrm{BaO}$ & - & $<15$ & 5 \\
\hline & Others & Arsenic oxide $<5$ & Arsenic trioxide $<0.5$ & Boron oxide 3 \\
\hline \multicolumn{2}{|c|}{ Refractive index } & 1.71 & 1.76 & 1.71 \\
\hline \multicolumn{2}{|c|}{ Knoop hardness $\left[\mathrm{kg} / \mathrm{mm}^{2}\right]$} & 370 & 440 & 370 \\
\hline \multicolumn{2}{|c|}{ Density $\left[\mathrm{g} / \mathrm{cm}^{3}\right]$} & 4.46 & 4.8 & $<4.36$ \\
\hline \multicolumn{2}{|c|}{ Transmittance [\%] (wavelength 550 nm) } & 86 & $<85.0$ & 88 \\
\hline
\end{tabular}

3.045 for potassium for a photon energy of $30 \mathrm{keV}$ ) [6]. Therefore, for the same lead equivalent, the glass thickness can be made thinner, and in fact, on passed to a ZFx thickness of $10 \mathrm{~mm}$, the thickness of MedX and LX-57B is $7 \mathrm{~mm}$. However, this difference in thickness is not thought to be a disadvantage when the glass is installed in a medical setting. The mass per unit area $\left[\mathrm{kg} / \mathrm{m}^{2}\right]$ is 37.3 for $\mathrm{ZFx}, 32.8$ for MedX, and 30.1 for LX-57B. Because MedX contains 20\% barium, the mass per unit area appeared to be larger than that of ZFx, but when the mole ratio of barium to potassium is considered, the mass of ZFx is seen to be larger. Also, because LX-57B is 5\% barium oxide, its mass is the lowest.

\subsection{Absorbance of Non-Irradiated Lead Glass}

According to IEC 61331-2, it is stipulated that the transmittance of light with a wavelength of $550 \mathrm{~nm}$ should be above $80 \%$ (but more than $85 \%$ is desired), and for a wavelength range 500 - $600 \mathrm{~nm}$, it must have basically the same transmittance.

Here, for an incident light intensity $I_{0}$ and a transmitted light intensity $I$, the relation between the transmittance $T[\%]$ and the absorbance $A$ is given by the following equations:

$$
T=100 \frac{I}{I_{0}}, \quad A=\ln \frac{100}{T} \rightarrow T=100 \cdot \mathrm{e}^{-A}
$$

Also, when the contact between two non-absorbing isotropic media 1 and 2, is in the form of a plane, for respective refractive indices of $n_{1}, n_{2}$, angles of incidence and reflection $\theta_{1}$, and angle of refraction $\theta_{2}$, then Snell's Law applies and $n_{1} \sin \theta_{1}=n_{2} \sin \theta_{2}$. The reflectance depends on the deflection direction and the angle of incidence of the incident light, but the reflectance $R$ [\%] and transmittance $T$ [\%] (intensity) for the case when it is incident perpendicularly $\left(\theta_{1}=\theta_{2}=0^{\circ}\right)$ is expressed by the following Fresnel's reflection formula:

$$
R=100 \cdot\left|\frac{n_{2}-n_{1}}{n_{2}+n_{1}}\right|^{2}, T=100-R
$$

For example, assuming $n_{1}=1.0$ (air), and $n_{2} \sim 1.7$ (glass), then $R \sim 6.72 \%$. When the light passes through the glass, there is no absorption or scattering, and including reflection from the underside as well, the transmittance of this glass has a maximum of around $87 \%$.

Figure 1 shows the absorbance of each different lead-glass type when it is not irradiated. Because the absorbance of all the lead glass at a wavelength of $550 \mathrm{~nm}$ is below 0.1 , the transmittance is then more than $90.5 \%$, which can be seen as satisfying the IEC 61331-2 stipulation given above. Furthermore, it can be seen that all lead grass has roughly the same transmittance for the wavelength range of $500-600 \mathrm{~nm}$. In particular, ZFx lead glass has a low absorbance compared to the other type of lead glass, and this shows that the transmittance of ZFx is high.

The MedX and LX-57B lead glass had a visual appearance of being tinged with yellow. This derives from the 


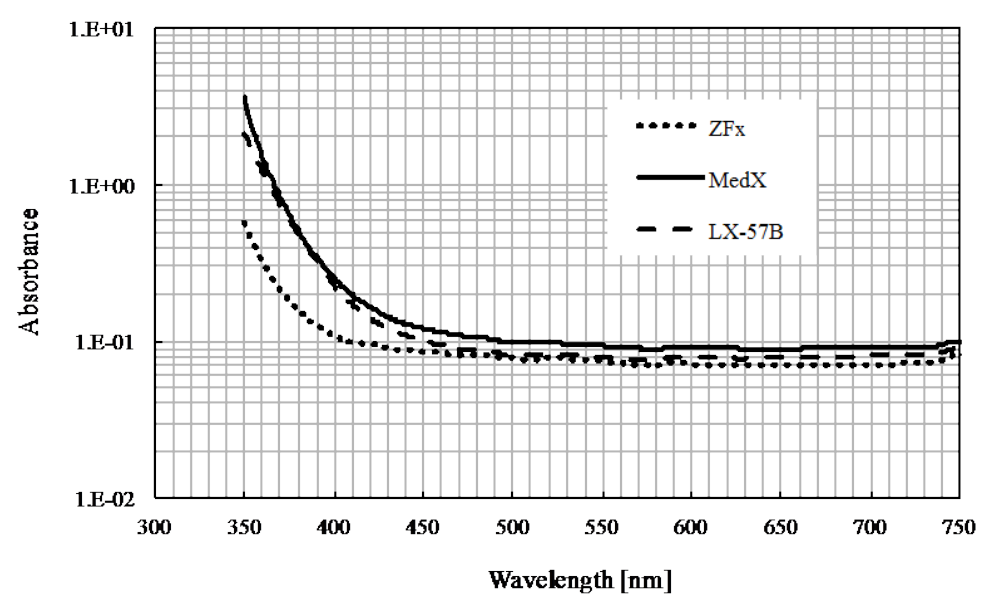

Figure 1. Absorbance for different type of non-irradiated lead glass.

fact that many dielectrics (insulators), including glass, are transparent for the region that includes visible light with no absorption, but on the short wavelength side of that (the ultraviolet region), there is absorption from electronic excitation, and on the long wavelength side (infrared region), there is absorption due to vibrational excitation, so that electromagnetic waves are not transmitted [7]. For example, silica glass shows a strong absorption at around $200 \mathrm{~nm}$ in the ultraviolet region and at around $3000 \mathrm{~nm}$ in the infrared region [8] [9]. In particular, the absorption edge of the ultraviolet region is determined by the band structure originating from the molecular orbital of the dielectric. Heavy metal low-valent ions, such as $\mathrm{Pb}^{2+}$ and $\mathrm{Ba}^{2+}$, are added to glass as an $\mathrm{X}$-ray protective material as in the case of lead glass. Because the $n s$ orbit occupied by the lone electron pair of these ions forms an energy level on the energy side higher than the 2p orbit of the non-bridging oxygen, the energy gap gets smaller, and the absorption edge wavelength shifts to the long wavelength side. Therefore, for lead glass with added $\mathrm{Pb}^{2+}$ and $\mathrm{Ba}^{2+}$, as the bottom of the ultraviolet absorption edge reaches visible light, it is colored either a pale yellow or yellow color. Nevertheless, ZFx does not visually appear yellowish despite the addition of $\mathrm{Pb}^{2+}$. It is thought that, with the addition of an alkali oxide, the energy level returns to its original state because its $n p$ orbit forms a hybrid orbit with the $n$ s orbit of the above-mentioned heavy metal ion, and the energy gap then becomes larger.

\subsection{Coloration with Increase in Absorbed Dose}

Figure 2, with absorbance at $550 \mathrm{~nm}$ along the vertical axis, and the logarithm of the absorbed dose along the horizontal axis, shows the absorbance curves for increases in the absorbed dose for each type of lead glass. These regression curves using logarithmic regression fit well $\left(R^{2}=0.87\right.$ for ZFx, $R^{2}=0.92$ for MedX, and $R^{2}=0.97$ for LX-57B), and can thus be thought to represent the basic trend in the curves. Because an irradiation time of 100 seconds is equivalent to a period of use of 1 year, it is evaluated for use up to 50 years. As the absorption curves for all the different types of lead glass have similar shapes, Figure 3 is given for ZFx as an example of an absorption curves for increasing absorbed dose. From the figure, the trend to increase can be seen for the wavelength region less than $650 \mathrm{~nm}$. This is because the absorption positions for the color center are at approximately 430 and $620 \mathrm{~nm}$. The glass is colored brown with increasing absorbed doses, a fact which corresponds with the increased absorbance in the low-wavelength region. As other products show the same rate of increase, the transparency of ZFx can be thought to be excellent compared to other products because, as described in Section 3.2, the absorbance below $450 \mathrm{~nm}$ for ZFx is low.

\subsection{Color Fading with Elapsed Time}

Figure 4 shows the absorption curve for ZFx measured over the wavelength range of 300 - $900 \mathrm{~nm}$ with an absorptiometer following continuous irradiation for 15 hours, from immediately after irradiation (after 5 minutes) to approximately 3 days later (after 4650 minutes). As with the results in Section 3.3, the absorbance is seen to decrease significantly for the wavelength region below $650 \mathrm{~nm}$.

The absorbance was measured for other types of lead glass as well, but as presenting a comparison is cum- 


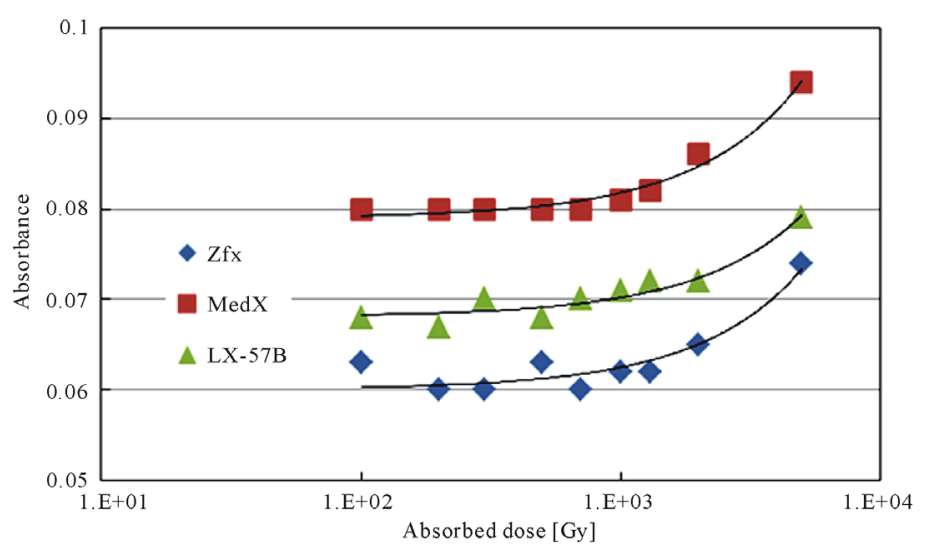

Figure 2. Absorbance $(550 \mathrm{~nm})$ for different type of lead glass with different absorbed doses.

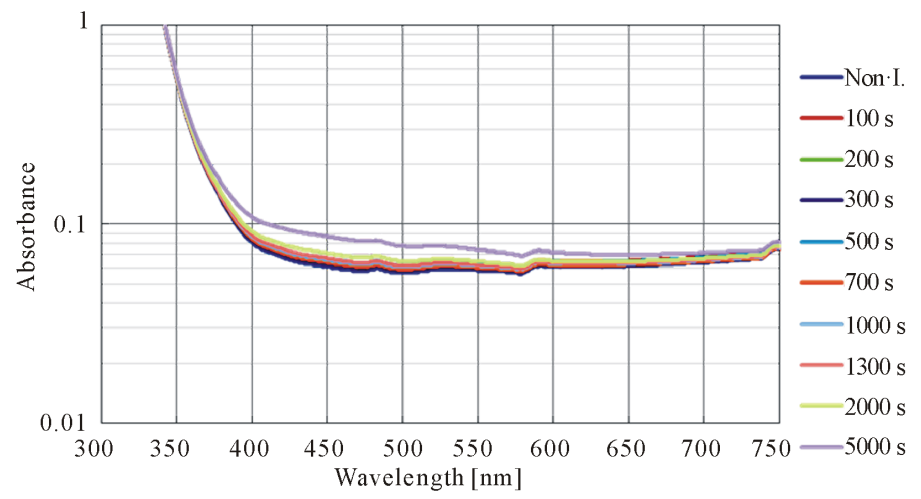

Figure 3. Absorbance curves with increasing absorbed dose (ZFx). Parameter shows irradiation time, and Non-I. is non-irradiation glass. Absorbance is increasing with the increase of irradiation time.

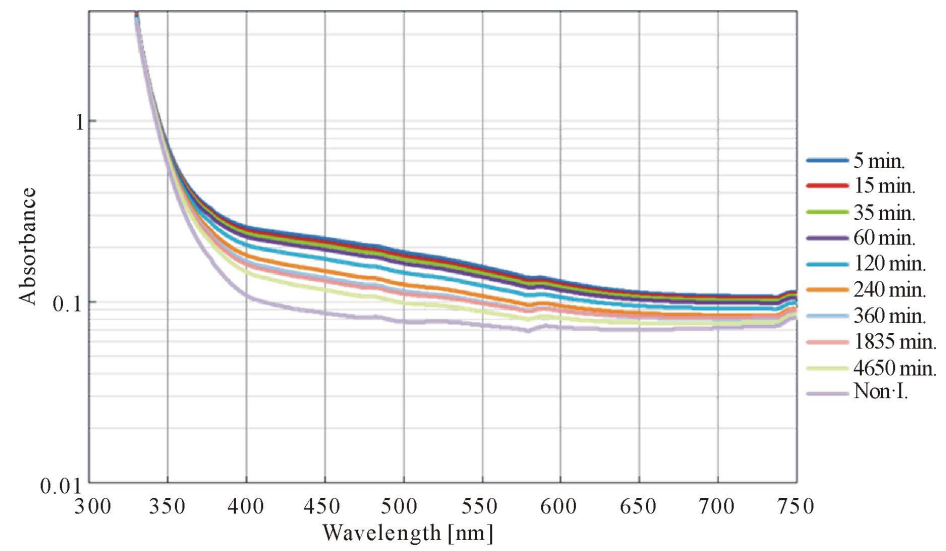

Figure 4. Absorption curves with elapsed time (ZFx). Parameter represents the elapsed time from the end of the irradiation, Non-I. was displayed as a reference in the non-irradiated glass. Absorbance with time is lowered.

bersome, Figure 5 simply shows changes in the discoloration over time for a wavelength of $450 \mathrm{~nm}$. The absorbance decay curves at a specific wavelength for all of the lead glass types are similar. However, ZFx and MedX have roughly similar curves, but because the absorbance for LX-57B is large, it forms a curve shifted in a parallel direction. Also, although the non-irradiated ZFx has a smaller absorbance than other types of lead glass, 


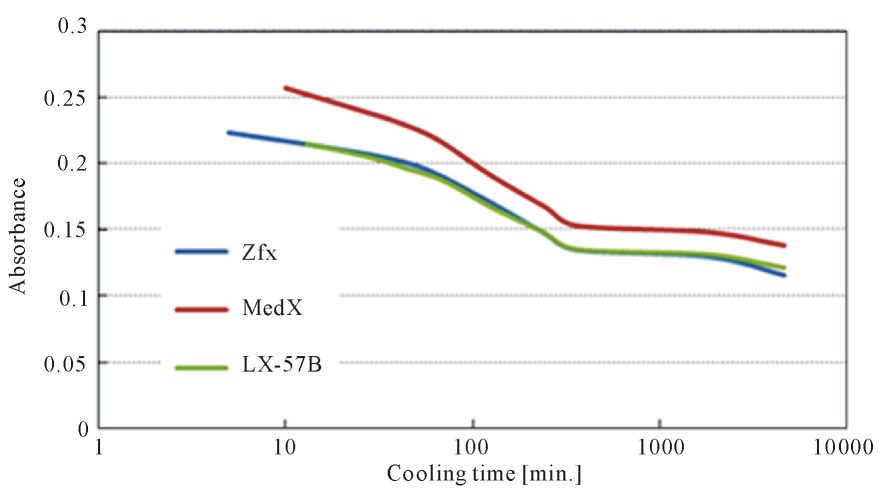

Figure 5. Variation of absorbance with elapsed time for different type of lead glass (wavelength $450 \mathrm{~nm}$ ). Curve of absorbance of each lead glass has been similar, ZFx and LX-57B was substantially the same curve.

we find that it is colored roughly similarly to MedX after being irradiated for 15 hours. In addition, from the figure, given the fact that a deflection point is seen at an elapsed time near 300 minutes for all of the types of lead glass, it would seem that the mechanism involved in the decoloration includes a component which has short and long half-life. Thus, dividing the elapsed time into two components of 0 - 300 minutes and 300 - 4650 minutes, we found an approximately straight line for each exponential function. As a result, the half-life of the short component for ZFx and MedX was 27.4 hours (coefficient of determination; $R^{2}=0.98$ ), and the half-life of the long component was 107 days $\left(R^{2}=0.99\right)$. The long component of LX-57B was 107 days for ZFx and MedX, but the half-life of the short component was 28.6 hours $\left(R^{2}=0.98\right)$.

\section{Conclusion}

Using an absorptiometer for lead glass from three different companies, we evaluated the transparency, the coloration due to X-rays, and the decoloration with elapsed time. The transparency of ZFx was high in comparison with the glass from other companies. In particular, the transparency was excellent below $450 \mathrm{~nm}$, and although visually, those of other companies had a yellowish color, it could hardly be discerned for ZFx. However, all three companies complied with the IEC 61331-2 regulations without any problems. But to guarantee the equivalent amount of lead, the thickness of ZFx compared with other companies was 1.4 times the thickness. The coloring due to X-rays, because the absorption positions for the color center were near 430 and $620 \mathrm{~nm}$, was seen to increase for the wavelength region below $650 \mathrm{~nm}$. The mechanism involved in the decoloration can be considered as having a component with short half-life, and a similar component with a long half-life, and whereas the half-life for the short component was about 28 hours, the half-life for the long component was 107 days.

\section{References}

[1] National Council of Radiation Protection and Measurements (2004) NCRP Report No. 145, Radiation Protection in Dentistry.

[2] National Council of Radiation Protection and Measurements (2005) NCRP Report No. 147, Structural Shielding Design for Medical X-Ray Imaging Facilities.

[3] International Electrotechnical Commission (2011) IEC 61331-2 Protective Devices against Diagnostic Medical XRadiation-Part 2, Translucent Protective Plates.

[4] Kakuno, K. (2009) Lectures for Newcomers and Leaders: Optical Properties of Glasses (2). New Glass, 24, 66-72.

[5] Uhlmann, D.R., et al., Eds. (1991) Optical Properties of Glass. The American Ceramic Society, Inc., New York.

[6] Maekoshi, H. (1995) Supervision Radiological Technology Studies Library (11), Photon Attenuation Coefficient Datebook. Japanese Society of Radiological Technology, Kyoto.

[7] Kakuno, K. (2009) Lectures for Newcomers and Leaders: Optical Properties of Glasses (1). New Glass, 24, 59-63.

[8] NEW GLASS Handbook Editing Committee Edition (1991) NEW GLASS Handbook. Maruzen Co., Ltd., Tokyo.

[9] Aggarwal, I.D., et al., Eds. (1991) Fluoride Glass Fiber Optics. Academic Press, Inc., New York. 